\title{
Biomechanical Approach to Improve the Abdominal Aortic Aneurysm (AAA) Rupture Risk Prediction
}

\author{
Guillermo Vilalta, Félix Nieto, Enrique San Norberto, \\ María Ángeles Pérez, José A. Vilalta and Carlos Vaquero
}

Additional information is available at the end of the chapter

http://dx.doi.org/10.5772/46030

\section{Introduction}

It is well known that the human body operates under a continuous interaction of complex processes taking place at multiple dimensional and temporal scales. While biomedical research is slowly elucidating many of these processes, it remains mostly unclear how they interact in the production of the global physiological or pathological conditions we observe [1]. The cardiovascular system in general and the Abdominal Aortic Aneurysms (AAA) in particular is a good example.

Aneurysm is a pathology that can affect most blood vessels, arteries or veins, and it commonly occurs in the cerebral vasculature and the thoracic aorta even if the vast majority of cases occur in the abdominal aorta and are termed AAA.

In its most accepted definition, AAA is a localized, progressive and permanent dilation (usually larger than $3 \mathrm{~cm}$ in diameter) of the aortic wall. Under specific conditions mainly associated with an irreversible pathological remodelling of arterial connective tissue, the aneurysm tends to increase in size, with an increased risk of rupture which can cause death. Atherosclerosis is the most common cause of aortic aneurysm. However the causes are usually multifactorial: environmental, genetic, autoimmune or infectious.

AAA has increasingly been recognized as an important health problem in the last decades. The statistics associated with this pathology are the major concern: AAA has been estimated to occur in $3-9 \%$ of the population [2], with a mortality rate on rupture between $78-94 \%$ [3] producing more than 15,000 deaths annually in the US and 8,000 in England. The mean age of patients with AAA is 67 years and men are affected more than women by a ratio 4:1 with prevalence up to $5 \%$ [4]. 
The majority of studies found in medical literature report this increase in the incidence of aortic aneurismal disease, which is expected in a continuously aging population in developed countries. In spite of significant improvement in surgical procedures and technological advancements in imaging devices in recent years, the associated aneurysm mortality and morbidity rate have also risen concomitantly.

Currently, the lack of an accurate AAA rupture risk index remains an important problem in the clinical management of the disease. The main clinical criteria in deciding on the treatment of AAA patients are: a) the peak transverse diameter and b) the growth rate. If the peak diameter reaches the upper threshold $(5-5.5 \mathrm{~cm})$ or the maximum diameter expansion rate is $>0.5 \mathrm{~cm} / \mathrm{yr}$ for smaller AAAs the patient may be submitted for surgical intervention, also depending on the state of health and willingness of the patients. The main limitation of this practice is that these criteria, although have a significant empirical basis, can be considered insufficient because they have not a physically sound theoretical basis. This statement should not be surprising; approximately 33\% of ruptured AAAs have diameters smaller than $50 \mathrm{~mm}$ [5] which is indicative of the complex pathogenesis of the disease progression that cannot be capture by traditional indicators.

Due to these observations, recently researches have been focused at improving the knowledge and the understanding of the phenomena associated with the formation and evolution of aneurysm pathology in order to define whether other variables could be predictive of rupture. The literature begins to reflect the existence of a consensus that, rather than empirical criteria, the develop of a biomechanical approach based on a multiscale model can be a significant step for the accurate assessment of the rupture risk.

This chapter examines the basis of the biomechanical approach. The main aim is to support the hypothesis that biomechanical considerations may become into powerful tool for a reliable patient-specific prediction of AAA rupture risk.

\section{Biomechanical approach. Method grounds}

This new approach has its foundation in the integration, through appropriate relations, of factors from different natures (biological, structural and geometric) and scales (temporal and dimensional) at the molecular, cellular, tissue and organ levels (from bottom level to top level), which allow to describe, from quantitatively point of view, the aneurysm progression and its rupture potential.

These defined relations are known as biomechanical factors or biomechanical determinants (BDs).

The basic premise of the biomechanical approach to estimate the AAA rupture risk, is that this phenomenon follows the principle of material failure, that is, an aneurysm ruptures when the stresses acting on the arterial wall exceeding its failure strength, reflecting the interaction between the arterial wall structural remodelling and the forces generated by blood flow within the AAA. 


\section{Remodeling history model. Biological Biodeterminants, BBDs}

Most investigators would agree that the pathogenesis of the abdominal aortic aneurysm (AAA) is multifactorial. There appear to be environmental, genetic, autoimmune, inflammatory, and structural factors.

The term "atherosclerotic AAA" is misleading because it suggests that atherosclerosis is a necessary cause of AAA disease. While some patients with have atherosclerotic occlusive peripheral vascular disease, others have minimal atherosclerotic disease. For this reason, the Joint Committee of the Society for Vascular Surgery recommending that the term "nonspecific AAA" be used since 1991.

The definition of AAA has varied in the literature over the years, but all definitions have in common a specification of the degree of aortic dilatation. So, the definition is a permanent localized dilatation of an artery having at least a $50 \%$ increase in diameter compared with the expected normal diameter of the artery or of the diameter of the segment proximal to the dilatation. According to this definition, an infrarenal AAA could then be defined as $3.0 \mathrm{~cm}$ if $2.0 \mathrm{~cm}$ is the expected maximal diameter of the infrarenalaort in an individual of a specific body scale.

\section{Risk factors}

The four principal positive risk factors for AAA are smoking, age, male sex, and family history. While smoking clearly seems to be an environmental factor, issues related to addiction and dose-effect responses are doubtless modified by genetic influences. The three principal negative risk factors for AAA are diabetes, female sex, and African-American descent, all of which are genetically determined.

There is a more complicated relationship between plasma lipid levels and the risk of AAA. Blanchard et al [6], failed to show any correlation between cholesterol levels, low-density lipoprotein (LDL) or high-density lipoprotein (HDL) and aneurysm risk (Blanchard). However, it have been showed an increased risk in patients whose plasma cholesterol was high and a protective effect was seen in patients whose serum HDL was high [7]. Low serum HDL gave an increased risk of AAA [8].

There is some disagreement in the literature regarding the effect of hypertension on aneurysm risk. The American Veterans study represented the largest of its type and showed hypertension to be an independent risk factor. Taking medication for high blood pressure was a risk factor, whereas hypertension itself was significant in women. Tornwall and Blanchard both showed both systolic and diastolic hypertension to be risks [6]. A study of all men born in Malmo in the year 1914 failed to demonstrate hypertension as a risk factor at all [9]. Experimentally, AAAs artificially induced into hypertensive rats were found to grow larger than those in normotensives [10] and the dilatation correlated well with systolic pressure.

\section{Molecular genetics}

Epidemiologic review indicates an aneurysm gene expression that is typically delayed until at least the sixth decade. There is strong evidence for inherited predisposition, and possibly 
an association with generalized arteriomegaly. It have been demonstrated an incidence of $20 \%$ aortic aneurysms among first order relatives of aneurysm patients [11]. In [12] was showed genetic linkages, accounting for abdominal aneurysm formation in 50 families, who had clustering of the lesion in two or more first order relatives. Possibly, they possessed a common metabolic disorder affecting the arterial wall.

A retrospective study of hospital patients in Zimbabwe demonstrated a higher incidence of aneurysms among whites than Africans [13]. By using ultrasound screening of first degree relatives demonstrated aortic aneurysms in $20-30 \%$ of male siblings over 55 years of age [14]. Case reports of familial aneurysm disease in patients without connective tissue or vascular diseases add validity to the theory of genetic linkage. The occurrence of multiple aneurysms in individuals is consistent with a genetic foundation. Many authors suggest aneurysm disease is a systemic process. Frequently, patients suffer from generalized arteriomegaly; often this is accompanied by multiple aneurysms.

Several cross-linking defects have been associated with aneurysm formation. Tilsonstudied the biochemistry of a collagen component deficiency that predisposes to aneurysms [12]. They evaluated pyridine cross-linkages and found fewer cross-linkages per collagen molecule in human skin samples. This suggests a genetic basis for aneurysm disease. Experiments with sex-linked defects of collagen and elastin demonstrate the blotchy BLO allele. These models exhibit aortic aneurysms and diminished skin tensile strength. The pattern of expression indicates the trait is related to the $X$ chromosome. In [15] it was reviewed the literature and found clear evidence for an independent genetic defect in most AAAs. Their work centered on a genetic analysis of collagen genes. Genetic collagen defects causing architectural defects are established in osteogenesisimperfecta (type I collagen of bone) and chondrodysplasias (type II collagen of cartilage). New evidence implicates mutations in the type III procollagen gene in the pathogenesis of aneurysmal disease. Various mutations have been confirmed in studies of patients with type IV Ehlers-Danlos syndrome (EDS) [16].

Studies of patients with aneurysms clearly demonstrate family linkage, and the data strongly suggest a genetic defect. Statistical analysis supports a recessive inheritance pattern in approximately $10 \%$ of men who have aneurysms. Research in this area is active and implicates an autosomal diallelic major locus.

The two genes with the strongest supporting evidence of contribution to the genetic risk for AAA are the CDKN2BAS gene, also known as ANRIL, which encodes an antisense ribonucleic acid that regulates expression of the cyclin-dependent kinase inhibitors CDKN2A and CDKN2B, and DAB2IP, which encodes an inhibitor of cell growth and survival. Functional studies are now needed to establish the mechanisms by which theses genes contribute toward AAA pathogenesis [17].

\section{Structural pathophysiology}

\section{Atherosclerosis}

The traditional view of aneurysm formation is that arterial dilation is a consequence of degenerative atherosclerotic disease, which results in acquired wall weakness. The 
experienced vascular surgeon is well aware that peripheral arteriosclerosis and aneurysmal disease often coexist. Severe atherosclerotic calcification in the aortoiliac vessels presents a technical challenge in aneurysm surgery. Epidemiologic, radiographic, and histologic data support the association between aneurysm disease and atherosclerosis [18].

AAAs and atherosclerosis share many risk factors and frequently occur simultaneously. The frequency of aortic aneurysms closely parallels the prevalence of atherosclerosis; for example, the low abdominal aneurysm rate in Asia correlates with the decreased incidence of atherosclerosis. Radiographic and histopathologic studies support the link between atherosclerosis and aneurysms. Ultrasound screening of patients with peripheral vascular disease detects a $5.9 \%$ rate of AAA, double that of the general population [19]. Studies of patients suffering from coronary and carotid artery occlusive disease detect an aortic aneurysmal rate of 11-13.5\% [9]. Histologic evaluations of sections from aortic aneurysms show atherosclerotic changes and thinning of the media.

Pathophysiologic principles also support the concept that atherosclerosis contributes to aneurysm formation. Atherosclerotic plaques may obstruct nutrient diffusion from the lumen to the media. The needs of the media must then be supplied exclusively by vasa vasorum from the adventitia. However, this may be inadequate due to incomplete distribution of vasa vasorum throughout the human arterial system [20]. Aortic vasa vasorum usually arise from the renal arteries, accounting for the relative sparing of the perirenal aorta from aneurysm formation.

Structural changes induced by atherosclerosis may contribute to aneurysm formation. As atherosclerosis progresses in humans, friable type I collagen replaces native type III collagen [21].Thus, the architectural integrity of the vessel is impaired, leading to a predilection to aneurysm formation. An association between aortic aneurysms and atherosclerosis is not surprising since the geometry and hemodynamics of arterial dilation predispose to atherosclerosis formation. Aneurysms have increased in incidence, prevalence, and mortality over the last 30 years, while coronary artery and cerebrovascular diseases have not. The divergence of these diseases in prevalence and mortality indicates that while risk factors are shared, the development of aneurysm disease is not entirely explained by atherosclerosis.

Although the epidemiologic link between the two is strong, it is propose that occlusive atherosclerotic aortic disease and aortic aneurysmal disease are distinct entities [12]. This is based on the different characteristics of these groups including age of onset, male-female ratio, clinical course, and prognosis. Evidence found to correlate with the size and state of aneurysm indicates that aneurysms reflect a heterogeneous disease with multiple forms and etiologic factors.

Autoimmunity

Autoimmunity may precipitate the inflammatory cascade. Aneurysm aortic extract was studied and noted to contain large quantities of IgG. Further studies revealed that the IgG from AAA patients was present and reactive against various proteins present in the 
aneurysmal aorta [22]. One of the initial putative autoantigen extracts was an 80-kDa dimer, designated aortic aneurysm associated protein-40 (AAAP-40). AAAP-40 was reactive with $79 \%$ (11 of 14 ) of AAA IgG preparations, and 11\% ( 1 of 9 ) of controls ( $p=0.002$ ) (Gregory). Other autoantigens have subsequently been found, and are currently under investigation in our laboratory. Evidence continues to accumulate to support the notion that autoimmunity may play an important role in aneurysmal degeneration of the aorta. Some of these autoantigens are absent in the external iliac artery, perhaps explaining why this artery rarely becomes aneurismal.

Triggering of autoimmunity can be brought about by autoantigens or molecular mimics. For example, molecular mimicry may occur with cytomegalovirus and clone 1. Also, rabbit antibody against Treponemapallidumand herpes simplex have been shown to bind to the adventitial elastin-associated microfibrils. The putative autoantigen AAAP-40 has homologies with Treponemapallidumand herpes. The hypothesis is that there are epitopes in the microbial proteins that are similar to the AAAP-40, thereby triggering an autoimmune response. Tanaka et al [23] detected herpes simplex viral DNA in 12 of 44 AAA specimens, compared with 1 of 10 normal subjects.

\section{Inflammation}

The normal aorta has few inflammatory cells within in its wall. An influx of CD3+ cells and lymphocytes is seen in AAA tissues Although $66 \%$ of all lymphocytes in AAAs are in the adventitia, polyclonal B-lymphocytes are abundant in the media. IgG is elevated in AAA specimens. In [24] it was showed an inflammatory infiltrate in the adventitia in $68 \%$ of 156 AAA resection specimens examined retrospectively. Macrophages are found throughout the wall of AAA specimens. The macrophage Fc receptors regulate the secretion of proteinases by receptor specific mechanisms. Phagocytes produce proteinases such as elastase and collagenase. On the other hand, it have been implicated the collagenase, stromelysin, and gelatinase-B (MMP-1,3,9) in the destruction of the aorta matrix [25]. Cytokines are released by inflammatory cells and smooth muscle cells in the aorta. They are predominantly: interleukin 1 (IL-1), IL-6, IL-8, monocyte chemoattractant protein (MCP-1), tumor necrosis factor (TNF), and interferon (IFN). These cytokines, to varying degrees, cause MMP expression, TIMP reduction, induction of prostaglandin synthesis, lymphocyte proliferation, and chemotaxis. An autoimmune or inflammatory cascade, as proposed in some etiologies of AAAs, is perpetuated via the use of cytokines [26].

\section{Enzymatic degradation}

The elastin: collagen ratio has consistently been shown to be reduced in AAAs when ompared with normal aortas, leading to loss of elasticity and weakening of the aneurysmal wall. This may not be simply due to increased elastin degradation, as Minion et al. have shown that the total elastin content of the aneurismal wall may actually increase, but that the corresponding increase in collagen is much greater (Minion). Despite this evidence, there is little doubt that proteolysis plays an important role in aneurysm development. Aneurysmal disease differs from stenotic disease by the intensity of proteolytic activity within the extracellular matrix. The established association with chronic lung disease 
supports the argument that elastolysis is a major contributory factor, and indeed this is an area in which there has been much research. For some time, the cause of elastin degradation remained unknown, but even as early as 1980 when it was described increased collagenase activity [27]. In 1991, it was found a spectrum of collagenase activity in the aortic wall of both atherosclerotic and aneurysmal vessels ranging from 55-92 kDa [29].

Importantly, although the collagenase activity was limited, it increased dramatically when tissue inhibitors of metalloproteinases (TIMPs) were destroyed. In [30], it wasalso described the increased expression of a 92 kDagelatinase in AAAs when compared with both normal aortas and aorto-occlusive disease, and localized this to the area around infiltrating macrophages. This gelatinase is part of a family of zinc-dependent proteolytic enzymes, the matrix metalloproteinases (MMPs), now known as MMP9. In the same year, Freestone et al [31],further elucidated the relative amounts of both MMP9 and MMP2 by a combination of gelatinzymography and immunoblotting. This study demonstrated that the principal gelatinase in smaller aneurysms was MMP2, but that in larger aneurysms MMP9 predominated. McMillan et al [21],investigated mRNA levels for MMPs in AAAs and found that MMP9 was maximally expressed in moderate diameter $(5-6.9 \mathrm{~cm})$ rather than large $(>7$ $\mathrm{cm})$ or small $(<4 \mathrm{~cm})$ aneurysms. These findings suggested that whilst MMP9 was responsible for the rapid growth that was seen in this size of aneurysm, other enzymes were responsible for initiation and rupture. Pyoet al.'s paper elegantly proves a link between MMP9 and aneurysm pathogenesis by looking at the effect of inhibiting it both pharmacologically and by targeted gene disruption [32]. Mice that were deficient in the MMP9 gene failed to develop aneurysms as their wild-type counterparts did when subjected to elastase perfusion of the aorta. Bone marrow transplants from each group to the other reversed the response to elastase infusion, demonstrating that the expression of MMP9 by inflammatory cells is crucial to aneurysm development. Other MMPs have also been implicated in the development of AAAs, particularly MMP1 and MMP3. Vine and Powell also found immunoreactive MMP1 in extracts from AAAs (Vine). And more recently the expression of MMP3, as measured by reverse transcriptase polymerase chain reaction (rtPCR), was found to be elevated in AAAs when compared to aorto-occlusive disease.

Matrix metalloproteinase 13 is a recently described enzyme also known as collagenase- 3 and its expression is tightly regulated. Whilst MMP13 was not expressed at all in normal tissue, it was found in atherosclerotic disease and in significantly higher concentrations in AAAs. Expression was localized to medial smooth muscle cells in the aortic tissue, and could also be detected in human vascular smooth muscle cells in culture. Membrane type MMP1 (MT MMP1) is an activator of MMP2 and was found to be increased in aneurismal aorta when compared to normal or atherosclerotic aorta. Membrane type MMP1 was localized to aortic smooth muscle cells and macrophages in aneurysmal tissue by immunohistochemical analysis. The ability to activate MMP2 was confirmed by the addition of radiolabelled proMMP2, and determination of the subsequent amount of radiolabelled active MMP2. In vivo, the activity of MMPs is tightly controlled by their natural inhibitors, the TIMPs. In 2000, it was demonstrated that TIMP-1 bound to both the monomeric and dimeric forms of MMP9, whereas TIMP-2 bound only to the active form. Whilst it has been shown that the TIMPs are 
present in large quantities in AAAs, it has been suggested that itis an imbalance between MMPs and TIMPs that leads to the net increase in proteolysis seen. Tamarinaet al also showed that the TIMP: MMP ratio was actually decreased in AAAs, despite an absolute increase in TIMP levels [33].

Whilst there has been considerable work published in the area of collagenases and other metalloproteinases in AAAs, less is known about the role of serine proteases. Elastases of approximately 20-30 kDa have been demonstrated in the inner aspect of the media in AAAs. This elastase works best in the alkaline range, and is inhibited by $\alpha-1$ anti-trypsin. The fact that it is also inhibited by phenylmethylsulphonyl fluoride (PMSF) confirms that it is indeed a serine protease. Five distinct serine proteases have been separated by gel electrophoresis from aortic aneurysm tissue, suggesting there is a spectrum of enzymes at work. In addition to MMPs and serine proteases, there is also the cysteine protease group. These differ from serine proteases by the substitution of an Asn residue for an Asp in the catalytic triad. Cathepsins $\mathrm{S}$ and $\mathrm{K}$ are examples of this type of elastase and have been shown to be produced in abundance by smooth muscle cells in atheroma. They are inhibited by cystatin $C$, the expression of which is governed by a polymorphism of its signal peptide. As discussed previously, patients in whom the cathepsins were not inhibited displayed faster growing aneurysms.

AAA is a multifactorial disease with genetic risk factors and an immunologic component. Immune cells, including macrophages, neutrophils, mast cells, B- and T- lymphocytes, along with vascular smooth muscle cells and adventitial fibroblasts, produce cytokines and enzymes, promoting an inflammatory reaction, extracellular matrix degradation, and neovascularization. Among the different enzymes secreted by immune and stromal cells, matrix metalloproteinase (MMP)-2, MMP-9, MMP-12, cathepsins, and neutrophil elastase cause medial degeneration. Chymase causes smooth muscle cell apoptosis, and MMP-3, MMP-8, and MMP-13 cause adventitial collagen degradation, promoting abdominal aortic aneurysm rupture [34].

\section{Oxidative stress}

The action of reactive oxygen species has been implicated in the etiology of many disease processes. In particular, the effect of oxidative stress on many aspects of vascular biology has come under intense scrutiny over the past few years. The addition of antioxidants significantly reduced the activity of MMP9, whereas the addition of inhibitors of protein kinase $C$ had no effect. These results suggest that the increased proteolytic activity seen in the extracellular matrix in patients with diabetes mellitus is due, at least in part, to the effects of oxidation, and may help to explain a link between aneurysm formation and oxidative stress. A further series of aortic banding experiments have demonstrated that in areas of high pressure there is an up-regulation of endothelial nitric oxide synthase (eNOS) when compared with tissues downstream of the artificial coarctation [35].

Measuring nitrotyrosine in the same tissues gave some indication of the degree of nitric oxide breakdown and sequestration by reactive oxygen species. In the areas above the 
banding (heart, brain and thoracic aorta) the levels of nitrotyrosine were much higher than in areas not exposed to high pressures (distal aorta). The inactivation of nitric oxide due to oxidative damage in areas of high pressure is another indication of vascular endothelial dysfunction, which may contribute to the pathogenesis of aneurysms. Combining the in vitro elastase perfusion rat model of Anidjaret al [10], with modern cDNAmicroassay analysis,looked at the expression of 8799 genes in rats with induced aortic aneurysms, and compared them with genes expressed in rats that had undergone sham operations [36]. Using this technique they were able to identify over 200 genes whose expression had more than doubled in the aneurysm group. Significantly, this included many genes reflecting an increase in oxidative stress, notably hemeoxygenase, inducible nitric oxide synthase (iNOS), 12-lipoxygenase and heart cytochrome C oxidase, subunit VIa. Conversely, antioxidant genes such as superoxide dismutase, reduced NAD-cytochrome b-5 reductase and glutathione $S$ reductase werefound to be down-regulated. These two complementary findings both point to oxidative stress playing a major role in AAA development.

\section{Infection}

Infected aortic aneurysms are uncommon, and infrequently have their pathological features been described. Panneton and Edwards evaluated clinical and histopathologic features in patients undergoing surgical repair of infected aneurysms of the descending thoracic or abdominal aorta over a 24-year period [37]. The results showed that among cases with an identifiable causative organism, staphylococcus accounted for $30 \%$, streptococcus for $20 \%$, salmonella for $20 \%$, Escherichia coli for $15 \%$, and other organisms for $15 \%$.

During recent years, attention has been paid to the role of atypical bacterial infections, including Chlamydia and Helicobacter pylori, in the process of atherogenesis and arterial disease development. The reported rates of detection within atherosclerotic lesions by PCR vary widely. Regarding Chlamydia, several studies hypothesized this organism as a possible source of vascular disease, including carotid, coronary, and aortic pathology. Its role in the pathogenesis of aortic aneurysms, however, has been controversial. Sodecket al [38],investigated the presence of C. pneumoniae in 148 tissue samples excised from control and diseased aortas. DNA of C. pneumoniae, C. trachomatis and C. psittaci were assessed by highly sensitive and specific real time polymerase chain reaction (PCR). C. trachomatisDNA was detected in $1 / 65$ diseased patients and in none of 83 controls $(P=0.43)$. In a similar study, surgical specimens derived from aneurysm or aorta fragments were investigated for C. pneumoniae utilizing PCR. In asymptomatic aneurysms, DNA was found in 9 cases $(29 \%)$, and in ruptured aneurysms in 14 cases $(49 \%)$. In the control group, C. pneumoniae DNA was not detected in the aortic wall. Conflicting data has failed to show a clear relationship between chlamydia infection and aortic pathology.

Cytomegalovirus (CMV)-induced arterial disease has also been linked to aortic pathology. To further elucidate the mechanism by which CMV may promote atherosclerosis, Westphalet al.(Westhpal), studied the expression pattern of cellular inflammatory and proliferative signals in the aortic wall of CMV (+) and CMV (-) patients undergoing coronary artery bypass grafting (CABG). CMV-DNA in smooth muscle cells was thought to 
induce local growth factor expression as well as endothelial activation, both of which can promote the progression of atherosclerosis. Since traditional atherogenic risk factors increase the likelihood of aortic CMV manifestation, CMV may play a crucial role in mediating the progression of atherosclerosis. The persistent expression of CMV-gene in the vessel wall plays a role in the vascular cellular response, including progression of atherosclerosis or vasculitis in vivo. Kilicet al [39], performed PCR analysis to demonstrate the relationship between CMV and atheromathosis at the aortic wall. CMV DNA was found in $37.9 \%$ atherosclerotic and $32.7 \%$ non-atherosclerotic vascular wall specimens.

\section{Vitamin E deficiency}

Studies have pointed to an inverse relationship between vitamin E (a-tocopherol) levels and the incidence of arterial disease. Vitamin $\mathrm{E}$ is an important lipid-soluble antioxidant that localizes to the hydrophobic area of biologic membranes [40]. In terms of AAA, it is hypothesized that activated polymorphonuclear cells (PMNs) release proteinases which degrade the aortic wall matrix. These same PMNs would also release oxidative enzymes, generating toxic oxygen species such as hydrogen peroxide which would lead to lipid peroxidation. Vitamin $\mathrm{E}$ is considered a specific, though indirect, index of in vivo peroxidation. They also showed that a small group of AAA patients had decreased vitamin E levels but not decreased vitamin E/total lipid ratios compared with controls (coronary artery disease and normal patients). Accordingly, the AAA patients may be under increased oxidative stress (e.g., increased inflammation or PMN activation) but do not have decreased concentrations of plasma vitamin E carriers.

This analysis reveals how the biological information associated with AAA pathogenesis constitute the foundation on which can be defined the destructive remodeling of the aortic wall and its influence in AAA rupture.

\section{Morphological Biodeterminants, MBDs}

After its formation, the aneurysm trends to increase in size and change its shape as consequence of the arterial wall destructive remodeling. This phenomenon, which occurs along many years in asymptomatic way, characterizes the AAA morphology and morphometry. Aneurysm geometric characteristics have been reported to be a significant predictors of the tendency for expansion or subsequent risk of rupture [41, 42] and can be the deciding factors in the clinical management of the disease. The correlation of the rupture risk with the aneurysm geometry has been clearly depicted in cases of intracranial aneurysms, where various shape indices were proven to discriminate sufficiently between rupture and unrupture aneurysms.

For AAAs, a pioneer work to assess the rupture risk based using the biomechanical concept was recently presented [43]. The authors combined geometrical and structural factors to obtain a dimensionless severity parameter, from which, they could estimate the potential risk of a specific aneurysm in any stage of development. Later, this concept it was modified for only considering the main geometric parameters of the aneurysm which can be easily 
determined by computed axial tomography (CT) or magnetic resonance imaging (MRI) obtained during periodic check-up [44]. The basic idea of the method was to correlate the main simple geometric parameters of the aneurysm in order to obtain the morphologic biomechanical determinants, MBDs. This idea is supported by the hypothesis that the aneurysm shape is strongly related with its rupture potential. Here, it is important to take into consideration that this method is a baseline for the determination of a rupture risk predictor and that such a treatment decision must be made within a reasonable turnaround time. Therefore, the precision of the method should be smaller than the clinical scale of evolution of the pathology and justifies the utilization of the aneurysm morphology based on simple geometric parameters as a rupture risk predictor.

Figure 1 shows an AAA schematic representation where the simple geometric parameters involved in this method are defined. $D$ is the diameter at the plane of maximum diameter, $D_{L}$ is the lumen diameter, $L$ is the aneurysm length which is measured from proximal neck to distal neck, $L_{A}$ is the anterior length measured from point of intersection $\mathrm{O}$ to anterior wall and $L_{P}$ is the posterior length measured from point of intersection $\mathrm{O}$ to posterior wall. During the follow up treatment the current clinical practice establishes that only three parameters are controlled: sagittal and coronal maximum diameter and length.

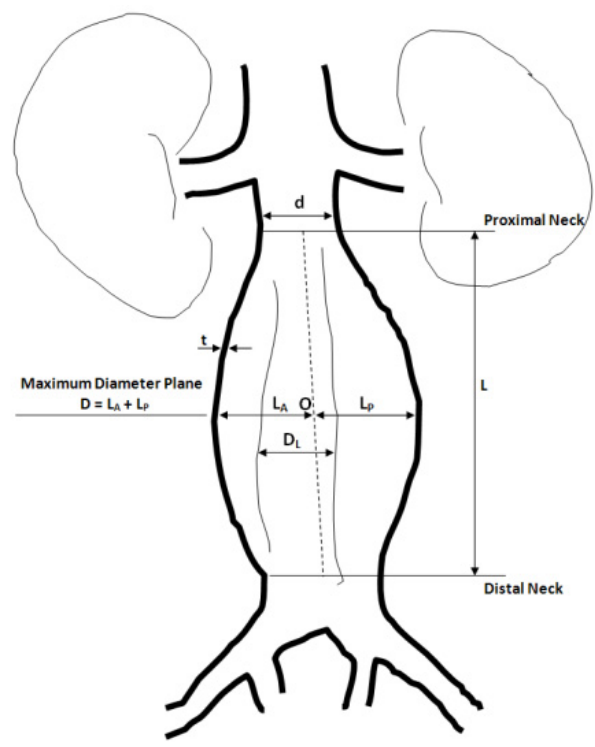

Figure 1. AAA schematic representation with the main geometric parameters.

After careful analysis, these simple parameters have adequately been combined to define the proposed geometric biomechanical factors. Some considerations about them are listed below:

1. Deformation Rate, $\chi$.Characterizes the actual deformation of the aorta. It is defined as a ratio between the maximum transverse diameter $D$ and infra-renal aorta diameter, $d$. 
This concept considers that the aorta diameters range between 1.5 and $2.5 \mathrm{~cm}$ for any patient. The value that defines a low rupture risk is taken as the lower deformation condition of the artery (lower values D and higher $\mathrm{d}$ ), and for the most critical condition, as the higher deformation (higher values $D$ and lower $d$ ).

2. Asymmetry, $\beta$.A characteristic feature of an aneurysm is its asymmetry, which can be attributed to the non-symmetry expansion of the aneurysm sac as a result of the expansionconstraints introduced by the proximity to the spinal column. Due to this, AAA geometry exhibits a high surface complexity and a significant tortuosity of the inflow conduit and the segments of the iliac arteries. An aneurysm has lower rupture risk if it is more symmetric $(\beta=1)$ and the risk increases as $L p$ tends to be lower than $L A$, which means that $\beta$ trend to 0 .

3. Saccular Index, $\gamma$. This factor assesses the portion of the aorta, with length $(L)$, which is affected by the formation and further development of the aneurysm. This means that long aneurysms have more rupture possibilities than a short one. Typical values of $L$ are ranged from 90 to $140 \mathrm{~mm}$ (some works have reported values of $L$, higher). The calculation condition of the upper threshold value is the higher value of $L$ and the peak value of $D$ (typical for elective repair).

4. Relative Thickness, $l$. The aneurysm geometric characterization determines the existence of a variable wall thickness; both between the anterior and posterior walls and between the aneurysmatic sac and the regions close to the distal and proximal ends. Initial studies have used uniform wall thickness in their attempt to characterize aneurysm shape. Although wall thickness was not one of the highest ranked features chosen with the feature selection algorithm based on the $\chi^{2}$-test, its effect on aneurysm rupture cannot be ignore [45]. Typical values of wall thickness $(t)$ in aneurysmatic arteries are ranged from 0.5 to $1.5 \mathrm{~mm}$ [46]. This general range may vary from $0.23 \mathrm{~mm}$ to $4.26 \mathrm{~mm}$ at a calcified site [47]. The danger of aneurysm rupture will be greater when the thickness is low in the peak diameter region. This trend falls with the increase of the wall thickness.

5. ILT/AAA area ratio, $\lambda$. Although $70 \%$ of AAA includes thrombus [48], there isnot consensus about its real influence in the AAA rupture phenomenon. Some investigators state that ILT may reduce the stress in the AAA wall, improving its compliance and significantly preventing AAA rupture. Other declared that ILT could accelerate AAA rupture. Hence, it is very important to consider the effects of ILT in the rupture potential, by means of the parameter ILT/AAA area ratio.

6. Growth rate, $\varepsilon$.It is considered as an important indicator for AAA rupture. A high expansion rate of $0.5-1.0 \mathrm{~cm} /$ year is often associated with a high risk of rupture, and an elective repair should be considered even if the maximum diameter is lower than $5 \mathrm{~cm}$. The value indicating that an aneurysm is in rupture risk has been determined regarding to the worst situation (the lowest value inside the range of high growth rate $(0.5 \mathrm{~cm} /$ year), the peak diameter $\mathrm{D}$ and the time $T$ between periodic check-up ( 0.5 year). The low rupture risk limits were determined for aneurysm formation conditions.

Once these factors were defined, it was necessary to evaluate their weight in the rupture phenomenon by means of the definition of the weighted coefficient $\omega i$ and of the weighted level risk WLRi. 
The weighted coefficient takes into consideration the weight of a specific factor on the frequency of occurrence of the AAA rupture. The initial values of the coefficients $\omega i$ have been obtained from the opinion of a group of surgeons about the importance of each factor. Furthermore, the weighted level risk considers the impact of a factor in the probability of AAA rupture and was sorted in four intervals: low impact, middle, high and dangerous. The WLR $i$ have been obtained from considerations made in open literature when the importance of a factor's value is given according to the level of risk.

Table 1 shows the threshold values assigned to each geometric biomechanical factor and their related weighted coefficient and level risk.

\begin{tabular}{ccccccc}
\hline \multirow{2}{*}{ MDDs } & Definition & $\begin{array}{c}\text { Low } \\
\text { Risk }\end{array}$ & $\begin{array}{c}\text { Middle } \\
\text { Risk }\end{array}$ & $\begin{array}{c}\text { High } \\
\text { Risk }\end{array}$ & Dangerous & $\begin{array}{c}\text { Weighted Coefficient, } \\
\omega i\end{array}$ \\
\hline Deformation Rate, $\chi$ & $\frac{D}{d}$ & $1.20-1.70$ & $1.71-2.30$ & $2.31-3.29$ & $\geq 3.3$ & 0.35 \\
Asymmetry, $\beta$ & $\frac{\left(D-L_{A}\right)}{L_{A}}$ & $1-0.9$ & $0.8-0.7$ & $0.6-0.5$ & $\leq 0.4$ & 0.10 \\
Saccular Index, $\gamma$ & $\frac{D}{L}$ & $\geq 0.75$ & $0.74-0.69$ & $0.68-0.61$ & $\leq 0.6$ & 0.10 \\
ILT/AAA ratio, $\lambda$ & $\frac{\left(D^{2}-D_{L}^{2}\right)}{D^{2}}$ & $0.1-0.24$ & $0.25-0.44$ & $0.45-0.61$ & $\geq 0.62$ & 0.10 \\
Relative Thickness, $l$ & $\frac{t}{D}$ & $0.05-0.04$ & $0.04-0.02$ & $0.02-0.11$ & $\leq 0.01$ & 0.10 \\
$\begin{array}{c}\text { Growth rate, } \varepsilon \\
\text { Weighted Level Risk, } \\
\text { WLRi }\end{array}$ & $\frac{\left(D_{A}-D_{P}\right)}{T}$ & $0.1-0.17$ & $0.18-0.3$ & $0.31-0.49$ & $\geq 0.5$ & 0.25 \\
\hline
\end{tabular}

Table 1. Geometric biomechanical factors characterization.

Hence, rupture risk quantitative indicator defined in term of AAA morphology, can be expressed as the sum of each weighted coefficient $\omega i$ multiplied by the corresponding WLRi:

$$
R I(t)=\sum_{1}^{6} \omega_{i} W L R_{1}
$$

Regarding the results of $R I(t)$, it is possible to advise several actions and suggestions to physicians. This is shown in Table 2.

As above indicated, the proposed method is based on six geometric biomechanical factors. But, it is possible that, for any reason, the information about some parameters is not available. In this case, the method fits its algorithm to calculate only the factors associated with the existing geometric parameters and it is able to weights the final result according to the amount of parameters taken into account.

An initial limitation of the method is associated with indirect errors in obtaining the MBDs, due to the difficulty in extracting exact values from the geometric parameters needed in determining these MBDs. The measurements of the simple geometric parameters is, usually, carried out by a radiologist, a human being with its professional customs and resources, 


\begin{tabular}{ll}
\hline $\mathrm{RI}(\mathrm{t})$ & Actions/Suggestions \\
\hline$<0.2$ & Rupture risk is very low. No action is suggested. \\
$0.2 \div 0.45$ & Rupture risk is low. A close observation is required. \\
$\div 0.7$ & Elective repair should be considered. Other symptoms such a back and \\
& $\begin{array}{l}\text { abdominal pain, syncope or vomiting, should be observed. } \\
>0.7\end{array}$ \\
\hline
\end{tabular}

Table 2. $R I(t)$ intervals and actions and suggestions offered by method to physicians.

with best and/or worst days, with/without personal and labor problems. Therefore, it is important to assess the influence of all these (and others) conditions on the precision of the results.

The ANSI-ASME PTC 85, ISSO 5167 standard was used to determine the indirect errors in the calculation of GBDs due to the direct measurements of the simple geometric parameters. The methodology was applied to data-base which was used for validation tests. The results that are shown in Table 3 correspond to higher values for the errors obtained. The bias limit in measuring of the geometric parameters for all parameters was considered $0.001 \mathrm{~m}$. The main conclusion that can be drawn from Table 3 is that the errors in determining the MBDs, are not significant.

\begin{tabular}{ccc}
\hline MDBs & Uncertainty, $U z$ & $\begin{array}{c}\text { Relative uncertainty, } \\
U(\%)\end{array}$ \\
\hline Deformation Rate, $\chi$ & $1.81 \mathrm{E}-01$ & 0.0464 \\
Asymmetry, $\beta$ & $2.55 \mathrm{E}-02$ & 0.075 \\
Saccular Index, $\gamma$ & $1.23 \mathrm{E}-02$ & 0.022 \\
ILT/AAA ratio, $\lambda$ & $1.81 \mathrm{E}-03$ & $3.13 \mathrm{E}-03$ \\
Relative Thickness, $\ell$ & $1.18 \mathrm{E}-02$ & 1.8 \\
Growth rate, $\varepsilon$ & $1.67 \mathrm{E}-02$ & 0.027 \\
\hline
\end{tabular}

Table 3. Indirect errors obtained in determining the GBDs.This standard allows defining the experimental uncertainty, $U$ in determining a variable $Z$, as:

This initial set of values was validated by using one clinical case and three cases from literature.

In shortly. In the clinical case, the state of a 74 year-old male patient with an aneurysm was assessed. The geometrical characterization shows that the peak diameter is lower than the threshold value $(50 \mathrm{~mm})$, therefore under current medical practice; the patient should be kept under observation. But, on the other hand, the values of the deformation rate and the asymmetry index fall into the high risk level interval. It must be noticed that by means of statistical analysis these geometric biomechanical factors are considered as the most influential factors on the aneurysm potential rupture.Other two MBDs are also sorted as high risk level, although their weight on the rupture phenomenon is lower. Therefore, the value of the patient-specific quantitative predictor calculated by equation (1) is $R I(t)=0.64$, which indicates that the elective repair should be considered. This result was confirmed 
because, during the period of check-up examination, the patient underwent an emergency surgical procedure for aneurysm rupture in the posterior wall.

In another test, a triple validation was performed comparing the results documented in the original papers [49], [50] and [51], the results presented by [43] and the results obtained with the proposed set of values [52]. The geometries of the different analyzed AAAs are very different, however the value of $\mathrm{RI}(\mathrm{t})$ is able to sort patients correctly. In the model presented in [49], it is noticed that the aneurysm affects a significant region of the aorta and has a high rate of growth, which has a high relative importance in the value of $R I(t)$. In the model [50], the two biomechanical factors that have more influence in the deterioration of the aneurysm increase in comparison with the previous one, but they stay in the range of elective repair, although it was expected that the indicator value would be higher.

Analyzing the model [51], it is noticed that there is a worsening of most of the geometric parameters; the most important are a high growth rate, a maximum diameter $20 \%$ greater than the threshold value and an aneurysm affecting a significant region of the artery. This behavior justifies that the value of the rupture risk indicator falls into the category of possible rupture.

These results encouraged the implementation of another validation test: a broader control study with a population of two hundred and one patients at the Clinic Hospital of Valladolid-Spain, who were submitted to Endovascular Aneurysm Repair (EVAR) treatment.Previously, a new the set of values for the weighted coefficient was defined by using a statistical tool to contrast the hypothesis that certain events have a probability of occurring. In this case, the event is associated to the AAA rupture due to a specific MBD.

According to this statistical tool, the new set of values resulting for $\omega i$ is: Deformation Rate= 0.35 , Asymmetry $=0.07$, Saccular Index $=0.1$, Relative Thickness $=0.07$, ILT/AAA area ratio $=0.07$ and Growth rate $=0.34$.

For this new test, the population of the sample was divided in three groups: Group I $(n=174)$ - patients without later consequences after EVAR treatment; Group II ( $n=5)$ - patients who died from causes associated with the AAA pathology; Group III $(n=22)$ - patients whose AAA ruptures. As all these patients were submitted to EVAR treatment, the main objective of this test is to verify if some of the surgical procedures in patients whose aneurysm has a maximum diameter higher than threshold value could have been avoided, and/or if the method can predict the rupture of aneurysm with a diameter less than the threshold value.

The results showed that in $88 \%$ of the patients who belongs to group I is justified the surgical procedure, because the $R I(t)$ values fall into dangerous and high level rupture risk. In the group II, the results suggest that the five patients should be submitted to surgical procedure because their rupture risk index is dangerous + high risk condition. All these patients died either during repair treatment or during recovering of it. The state of health of all these patients was not good, because they presented other diseases like renal chronic insufficiency, atheromatic plaque, previous complications related with cardiovascular diseases, digestive hemorrhages. 
Very interesting results are obtained in the analysis of the group III. The values of $R I(t)$ indicate that $95.4 \%$ of the patients, present levels of rupture risk sorted as dangerous and high and the surgical procedure could have been considered before rupture. All these patients had aneurysms whose maximum diameter was less than the threshold value for surgical treatment and a systematic (time between two consecutive revisions lower than 1 year) follow-up check are suggested to diminishing the risks associated to emergency surgery by ruptures.

The fact that one patient presented a middle rupture index was somewhat unexpected and it is probably attributable to a combination of other factors not considered here, associated to factors of biological and/or structural nature. It was verified that the geometric parameters are lower than the threshold values.

The obtained outcomes are promising and have motivated further actions. Recent studies [53] have identified other MBDs based on the lumen centerline geometry. According to [54], the resulting centerline is a piecewise linear line defined on the Voronoi diagram, whose vertices lie on Voronoi polygon boundaries [55]. Values of Voronoi sphere radius $R(\mathbf{x})$ are therefore defined on centerlines, so that centerline points are associated with maximal inscribed spheres. Since centerlines were constructed to lie on local maxima of distance from the boundary, there is a tight connection between maximal sphere radius and minimum projection diameter used in clinical evaluation. In fact, classic angiographic vessel diameter evaluation is performed considering the minimum diameter obtained by measurements on different projections. The availability of a robust method for centerline computation and diameter measurement allows to characterize blood vessel geometry in a synthetic way, therefore giving the opportunity of performing a study on a population of models. Since it has been shown that planarity, tortuosity and branching angles have a major influence on complex blood flow patterns, such a study may reveal if particular vessel configurations are involved in vascular pathology.

Three MBDs have been defined using this approach: tortuosity, curvature and torsion centerline. Today, VMTK software havebeen developed to 3D reconstruction of the lumen centerline geometry. Figure 2 shows the visual representation of these determinants. Tortuosity, an absolute number, expresses the fractional increase in length of a tortuous vessel in relation to the imaginary straight line and has been described in [55]. Torsion is measured in $1 / \mathrm{cm}^{2}$ and curvature is measured in $1 / \mathrm{cm}$.
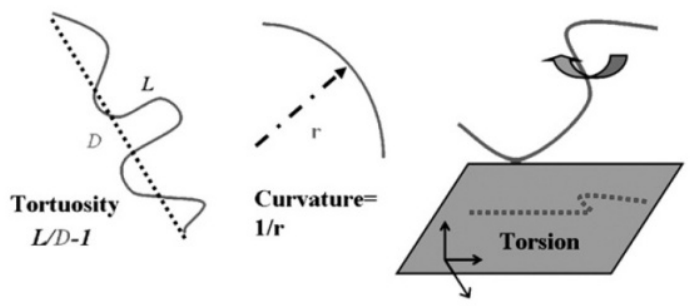

Figure 2. Schematic visualization of tortuosity, curvature and torsion [53]. 
Recently, it has been postulated that aneurysm peak wall stress (PWS) may be superior to diameter as predictor of the rupture risk. This statement has its theoretical foundation in the physical principle of the aneurysm rupture. Complex AAA geometry contributes to equivalent complex wall stress distribution over the entire AAA, with the higher stresses associated with regions of high curvature [56].

The role of these geometric biodeterminants in the prediction of AAA it has been assessed taking into consideration the presence of intra-luminal thrombus (ILT) [53]. In the study were included nineteen patients whose-which AAA maximum diameters ranged from 5 to $12 \mathrm{~cm}$. Statistical analysis confirmed that the maximum diameter significantly influenced PWS and the tortuosity may also affect PWS values in models with ILT in the same direction.

On the other hand, it has been demonstrated [57] that PWS is strongly correlated with the maximum diameter as well as the centreline asymmetry. It is notable, however, that in $73 \%$ of the analyzed models in this work a significant correlation was found between asymmetry and maximum diameter. Therefore, if diameter strongly correlated with peak stress then asymmetry would also score high.

Perhaps one of the most ambiguous issues in the assessment of rupture risk is the existence as well as the develop of the ILT. Despite ILT's impact on aneurysm disease, little is known about its development, and it is unclear whether it increases or decreases the risk of aneurysm rupture. That is, the ILT reinforces proteolytic activity [58], which weakens the wall [59], or buffers against wall stress [50]. It has been hypothesized that ILT develops either from rupture of vulnerable plaques or as a more continuous process characterized by blood-flow induced activation of platelets and their deposition at non-endothelialized sites of the wall exposed to low (sub-physiological) wall shear stress [60].

Recently, the investigationsare addressed to the integration of ILT in the computational models and, consequently, its effects in patient-specific on PWS values and distribution. A significant difference in PWS when including the ILT in 3D AAA computational model it has been reported [61]. Wang et al [50] showed that computational integration of ILT in 3D models could actually modify not only the value but also the distribution of PWS, thus playing a protective role against rupture but this conclusion was not supported in [62]. On the other hand, there is still some concern regarding the protective role of ILT, since many authors who evaluate the influence of ILT on hemodynamic stress transmission, reported that he presence of ILT fails to reduce the transmission of this stress on the AAA wall, consequently, leaving the AAA rupture risk equalled [63]. AAAs can experience higher stresses at regions of inflection, regardless of wall thickness variation. In such cases, the concentric or eccentric location of ILT in the AAA sac cannot be effectively reduce PWS values or changes its distribution [64]. A question of interest arises here, regarding whether such PWS values derived from computational estimation should be taken into consideration, since AAA rupture rarely takes places at these sites, reserving this possibility only for thrombosed AAAs [65]. Therefore, all these ideas reinforce the need to quantify and take into consideration the effect of the ILT. 
Finally, it is important to address the topic related to the use either simple geometric parameters or biodeterminants in the AAA rupture risk assessment. To answer this question some aspects should be analyzed. The first one is related to the temporal scales of the disease progression which is higher than the results' precision in the determination of the geometric parameters. This conclusion justify the use of morphological determinants. On the other hand, is the fact that aneurysm shape has a significant influence on flow patterns and consequently in its rupture potential. Recent findings have shown that the aneurysm geometrical shape may be related to the rupture risk. The morphological nature determinants (MBDs) are defined by appropriate relations among simple geometric parameters to characterize the influence of the aneurysm morphology on its rupture potential.

Utilizing idealized aneurysm models of the true vessel lumen surface geometry, the role of the geometric characteristics in the hemodynamic stresses prediction by using of Pearson's rank correlation coefficients was assessed [66]. In this work, the model was modified to allow the parametrization of the main parameters assessed: maximum diameter $\mathrm{D}$, length $\mathrm{L}$ and asymmetry, $\beta$. Figure 3 , shows a schematic view of the models used in this study.

The results show that hemodynamics stresses correlate better with MBDs. For hemodynamic pressure, the relation with saccular index and deformation rate are strong and negative $(\mathrm{r}=-$ $0.75, \mathrm{p}=0.000$ and $\mathrm{r}=-0.7, \mathrm{p}=0.000$ respectively). The asymmetry coefficient has no-significant correlation $(\mathrm{r}=-0.25, \mathrm{p}=0.00)$.

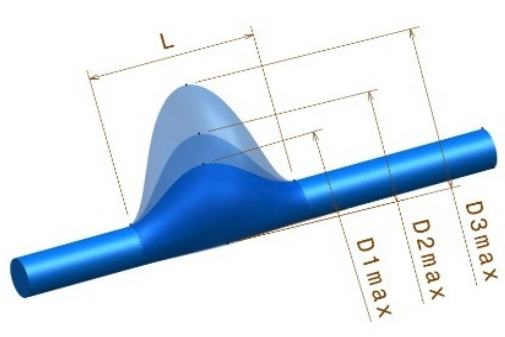

(a)

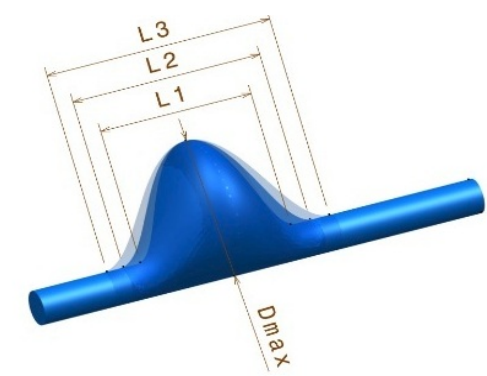

(b)

Figure 3. Schematic view of the parametrized aneurysm models. a) $L$ and $\beta$ are constant and $D$ varies; b) $D$ and $\beta$ are constant and $L$ varies.

The relation of asymmetry and deformation rate with WSS is weak with significance less than $15 \%$ and saccular index is no significant.

The main conclusions of this study are: luminal pressure is the primary mechanical load on the aneurismal wall and that MBDs are better predictors than simple parameters of the hemodynamic stresses.

On the other hand, Raghavanet al [67], showed that the deviation of the aneurysm shape from spherical configuration, the level of its surface ondulation or ellipticity and the norm of the surface mean curvature are a good predictors of rupture. 
This analysis confirms that MBDs may become a useful addition to current clinical criteria, mainly maximum diameter, in the decision-making process of the aneurysm treatment. Certainly, as in the same way that other biomechanical consideration, the suggested models require further studies.

\section{Structural Biodeterminants, SBDs}

In addition to morphological factors, numerically predicted wall stress, finite element analysis rupture index, rupture potential index and severity parameters have been proposed as alternative approaches to assessing rupture risk [68].

The criterion currently used by the medical community is that you can relate directly the risk of rupture with the maximum diameter of the aneurysm. However, as noted above, the biomechanics states that rupture occurs when wall stress exceeds its strength. This assumes a linear relationship between the maximum stress and the maximum diameter of the aneurysm. Thus, we propose an equation to describe this approach.

$$
\sigma_{\max }=k R_{\max }
$$

where $\sigma_{\max }$ is the maximum stress in the aneurysm, $k$ is a constant determined by experience, and $R_{\max }$ the maximum radius of the aneurysm. The maximum diameter criterion has many limitations.

Since there is currently no method to determine the stresses in the wall in vivo, it is necessary to develop models of the mechanical behavior of the arterial wall. These models can be generated from ideal parameterized geometries created by three-dimensional design software (CATIA, SolidWorks, etc.), or can be obtained through the processing of medical images.

Once the geometry is generated, we calculate using finite element method software (ANSYS, ABAQUS, etc.) in order to determine the stress distribution in the wall of AAA.

\section{Structural biomechanical determinant of VandeGeest}

After evaluating the stresses, and using the ultimate strength of arterial tissue or an assessment of the strength of the wall, you can define a structural biomechanical factor. This factor or biodeterminant, can allow us to estimate how close an aneurysm can be of the rupture and, consequently, the appropriateness of the surgical procedure in the patient.

Thus, it is proposed [69] the following factor:

$$
R I(t)=\frac{\text { Stress }_{i}}{\text { Strength }_{i}}
$$

whereiis the chosen point on aneurysm geometry.

It is noted that when the rupture index approaches the value of 1 , the state of risk of aneurysm rupture increases, ie when the stress observed in the wall reaches the value of strength. 
If the strength is only an estimated value for the entire aneurysm, use the maximum stress given by the simulation. But, when using the strength distribution in the whole geometry, the rupture index is evaluated at each point of the geometry of the aneurysm.

\section{Rupture criterion of Li and Kleinstreuer}

This approach [70] is based not only on statistical analysis of some cases of abdominal aortic aneurysms, but also on results of numerical simulations. To do so, tests were conducted with 10 patients whose data were known, in order to verify the accuracy criterion used to calculate $\sigma_{\max }$ :

$$
\sigma_{\max }=0.006 \frac{(1-0.68 \lambda) e^{\left(0.0123\left(0.85 P_{\text {sist }}+19.5 D\right)\right)}}{t^{0.63} \beta^{0.125}}
$$

where $\sigma_{m a x}$ is the maximum stress that appears frequently in an area whose diameter is equal to two thirds of the maximum diameter of AAA, $\lambda$ is the ratio of the areas in the plane of maximum diameter $\left(\lambda=A_{I L T, \max } / A_{A A A, \max }\right), \beta$ is the coefficient of asymmetry, Psis is the systolic blood pressure $(\mathrm{mmHg}), D$ is the maximum diameter of AAA $(\mathrm{cm})$ and $t$ is the thickness of the wall in the plane of maximum diameter.

If the thickness of the arterial wall cannot be determined from images taken by the TAC, can be approximated by the following equation:

$$
t=3.9\left(\frac{D}{2}\right)^{-0.2892}
$$

According to the authors, this approach presents a very low error in the determination of the maximum stress compared to other models. Whatever the feature is used to calculate stress, the results are very similar to the stress determined by finite element method software.

Clearly, the geometry should not be too complex, which is a limitation. Furthermore, the location of the maximum stress cannot determine, although the value is known.

This approach appears to be quite accurate results, and its application is very simple. So it could be used to determine the maximum stress of the aneurysm with a very simple approach. However, we emphasize that in no other study has been applied.

\section{Rupture criterion based on remodeling history model}

These models allow determining a stress value, which is compared with the strength of the arterial wall to evaluate if the break is close or not.

The value of strength can be obtained:

- Form literature, which are based on uni-axial tests aneurysmal tissue of patients.

- By an empirical approach based on an expression that takes into account the patient's personal information.

\section{Criteria based on two-dimensional modeling}

- It is a very simple model in two dimensions of the arterial wall; 
- $\quad$ The maximum stress is located at the maximum diameter;

- The aneurysm is cylindrical (or spherical);

- Wall thickness constant E;

- Linear elastic behavior.

From these criteria leads to a simple equation that relates the pressure $P$, the wall thickness $t$ and the maximum radius $R_{\max }$ of the aneurysm:

$$
\sigma_{\max }=P \frac{R_{\max }}{t}
$$

This modeling, which leads to the stress calculation, presents the following limitations:

- $\quad$ The geometry is very simple, which influences the results.

- Although you can adjust the value of the pressure acting on the wall, assigning the value at the studied patient's blood pressure, stress is always proportional to the radius of the aneurysm.

This approach is similar to the criterion of maximum diameter used today.

\section{Criteria based on three-dimensional modeling}

a. Modeling of material behavior: linear elasticity.

Many authors have used an elastic model of the arterial wall in their research [71, 72]

Commenting on the approach proposed in [73], the authors have attempted to determine the influence of the diameter and symmetry in the mechanical stress of the arterial wall of abdominal aortic aneurysm using an elastic behavior of the wall.

This approach has the merit of taking into account the behavior of the material used, and the authors are aware of the limits of their model, since the aim of their study was to show the influence of symmetry. However, other studies [74, 75] showed that the hyperelastic behavioral model is more suitable for simulating an aneurysm under pressure due to the large strains that can undergo aneurysmal arterial wall (20-40\%).

b. Modeling of material behavior: hyperelasticity.

Given the fact that the tissue of the aneurysmal arterial wall can be deformed the order of $20-40 \%$, the behavior can no longer be considered as elastic.

Hyperelastic materials are characterized by the existence of an energy function W, which depends on the state of deformation.

Tensions can be calculated with this energy function $W$, which depends on the material, which can be isotropic or anisotropic, which will influence in $W$.

b.1) Isotropic hyperelasticity

In 1940, Mooney and Rivlin established a behavioral model for the material like rubber, whose behavior is similar to the tissue of the arterial wall due to the incompressibility of both materials. 
Heng et al. [76], used the Mooney-Rivlin equation to establish one of the simplest hyperelastic models. The problem with this model with only two parameters is that is more suited to the study of polymers. This law was made by Mooney to model the behavior of rubbers, and it seems too simple for the study of tissues, whose behavior seems much more complex because its composition is not homogeneous.

You can also use a more complex form of Mooney-Rivlin model. In [77] it is performed a study which uses this model and the results seem that calculate appropriately the real tensions of the arterial wall. This form uses 9 parameters addition to the incompressibility parameter.

In 2000, it is defined a mathematical model using a regression from experimental results [75]. This is part of the theory of finite deformations and is based on the first principle of mechanics of continuous media. The assumptions underlying this model were that the wall is non-linear, homogeneous, incompressible and isotropic.

In 2006, this model is modified using another form of the density function [78]. It is observed that for incompressible materials considered, this equation is the same as proposed in [75].

In 2008, it is proposed a model based on the concept of material failure energy $\Phi$ [79]. This energy is the maximum amount of energy that the wall can withstand before breaking, because of the deformations. This value depends on the atomic or microscopic structure of the wall of an AAA.

b.2) Anisotropic hyperelasticity

- $\quad$ single transverse anisotropy.

In 1976, Tong and Fung [80], developed a cross-anisotropic hyperelastic model, which allows a behavioral model of the arterial wall aneurysm.

- Anisotropy with two families of fibers

- Rodríguez anisotropichyperelasticmodel [81];

- Holzapfel anisotropic hyperelastic model [82]. Proposed model for biological materials with two families of collagen fibers, as they really are the arterial walls.

The anisotropic hyperelastic behavior models better approximate the actual behavior of the aneurysmal arterial wall, but according to the model used, the results can be very different. One can see that the Rodríguez hyperelastic anisotropic model is closer (at the level of stress distribution) to an isotropic hyperelastic that the Holzapfelhyperelastic anisotropic model, as shown in Figure 4.

\section{c. Fluid-Structure Interaction}

All approaches that have been presented are based on the physical principle of fault material of aortic wall. However, all these approaches use a constant pressure value (often the peak systolic pressure), whereas, in reality, not only the pressure varies, but also the blood moves. In an attempt to make models as realistic as possible, we have developed the 
modeling fluid-structure interaction (FSI), in which the model considers simultaneously the effect of blood flow on the arterial wall and vice versa.

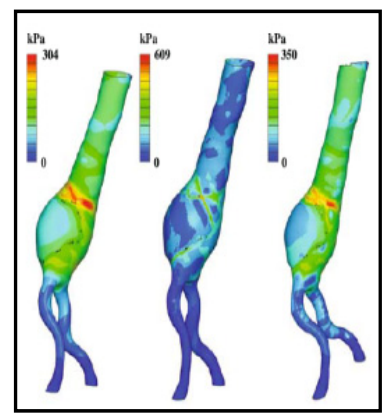

Isotropic Holzapfel Rodríguez

Figure 4. Stress in different models.

Some authors try to use a method of modeling of blood flow, to study its influence on the stresses of the aneurysm wall. These approaches are also used mechanical simulations to assess the stress in the wall of the aneurysm.

From the results obtained with FSI simulations [46], has been determined that in the simulations using the computational analysis of the static stress incurred in an underestimation of wall tension, which is shown in Figure 5 . This value can reach $12.5 \%$, as reported [83].

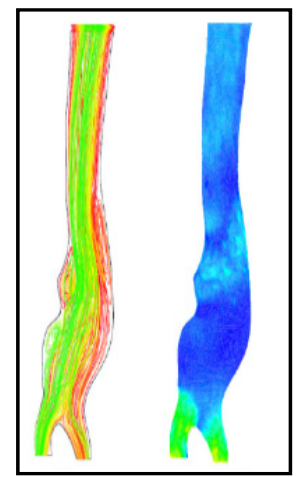

Figure 5. Stream lines which characterize the blood flow inside the aneurysm and surface distribution of stresses, obtained using a modeling FSI.

In 2006, a simulation of aneurysm under pressure [41] and blood flow was carried out in addition to demonstrating that when taking into consideration the bloodstream, the stresses change little while the time required for the simulation is three to four times greater.

The authors concluded that the fluid-structure interaction approach is interesting, but a modeling of the wall with the systolic pressure is sufficient to calculate the stresses in it. 
After the revision presented we can conclude that an anisotropic hyperelastic model, using systolic pressure load, and geometry with the important details of the AAA, is the best choice for calculating the stresses in aneurysmal wall.

Evaluation of the arterial wall strength.

At this point, is already known that the evaluation of the wall stress cannot be considered as an isolated indicator to assess the risk of rupture of AAAs, as an aneurysmal wall region which is subjected to high stresses, may also have a high strength, thus equalizing potential rupture. According to the remodeling history model, the strength of the wall is different from patient to patient and in the same patient at different regions and time scales. To resolve this situation, has been developed a technique for noninvasive estimation of the distribution of strength, defining a potential rupture index (RPI)[84], with equation:

$$
\text { Strength }=141.26-17.16 I L T+3.39 A G E-257.3 N O R D-69.5 H I S T
$$

where ILT is the thickness of the ILT (in cm), AGE is the patient age in years, NORD is the diameter normalized to the maximum diameter of AAA, HIST is $\pm 1 / 2$ according to family history $(1 / 2$ if the history is positive, $-1 / 2$ if no background) and SEX is $\pm 1 / 2$ by sex of the patient ( $1 / 2$ if the patient is a man, $-1 / 2$ for women).

These authors have increasingly improved this criterion, being the last, expressed by equation 8 , thatbest approximates the strength of the wall.

$$
\text { Strength }=72.9-33.5\left(\left(I L T^{0.5}\right)-0.79\right)-12.3(\text { NORD }-2.31)-24 H I S T+15 S E X
$$

\section{Rupture of aneurysm prediction}

The logical process for estimating the risk of aneurysm rupture using structural biomechanical factors would be the one described below:

1. Obtain the blood pressure of the patient.

2. CT of the patient's aneurysm.

3. Geometric model of the aneurysm from medical imaging.

4. Simulation of the aneurysm using data specific to the patient.

5. Estimating the strength of the arterial wall aneurysmal of the patient.

6. State estimation of risk of rupture of the aneurysm.

Subsequently, using the Rupture Index $(R I)$ proposed in Equation 1 can be estimated if a ruptured aneurysm is close. Obviously, it will require a medical evaluation of patient state of health (PSH).

\section{Simulation using the Finite Element Method (FEM)}

Unable to provide a method for determining the in vivo distribution of wall stress, nowadays it is used the finite element method (FEM), which is recognized as a very precise technique, which aims to find approximate solutions of partial differential equations and integral equations. Equations are solved at the nodes of the meshes that are generated and interpolated within the element, generating a continuous solution throughout the domain. 
Overall analysis by using Finite Element Method is an orderly process that will include the following steps:

1. Generation of geometry. The geometry can be generated or imported. In the case of aneurysm geometry is imported directly from the patient CT using some of the commercial software or open source currently available, so it has the actual geometry of the aneurysm affecting the patient under study. Figure 6, shows the geometric model of AAA obtained by the processing of medical images using the public software MeVisLab.

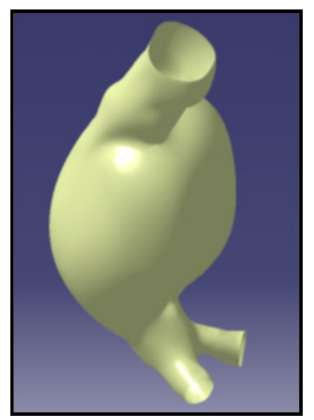

Figure 6. Geometric model of AAA obtained from the processing of medical images.

2. Discretization of meshing domain: The structure or part is divided into elements and modeled as a finite element mesh. In this step the analyst must decide the type, number, size and order of items to be used. This decision will characterize the degree of confidence results thereafter. An example that represents the arterial wall mesh is presented in Figure 7.

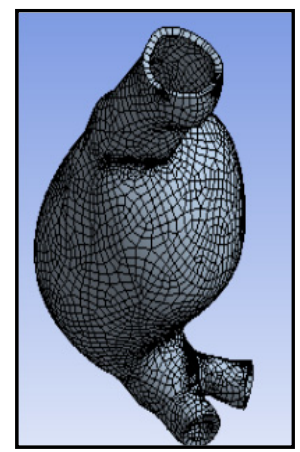

Figure 7. Mesh representation of a geometry that represents the arterial wall of an AAA.

3. Application of the boundary conditions: apply the loads which will be under the model (in this case the blood pressure) and the restrictions of the same (in this case is assumed to be attached to the remainder of the artery limiting their movement and must be taken into account if organs or body parts that limit their movement). 
4. Solution of unknown nodal displacements: The global balance equation is modified to take into account the boundary conditions of the problem and to obtain algebraic equations where the unknowns are nodal displacements.

5. Calculation of stresses and strains of the elements: Knowing the nodal displacements resulting from the previous stage, it could be calculated the stresses and strains using the corresponding mechanical equations.

6. Evaluation of results: the stresses solutions are obtained (and displacements in some models) along theaneurysm. It is possible to locate the exact point of the aneurysm where it produces the maximum stress and the value thereof. Figure 8 , shows the surface distribution of stresses. The red color indicates the region with higher values of stress and therefore, with greater risk of rupture.

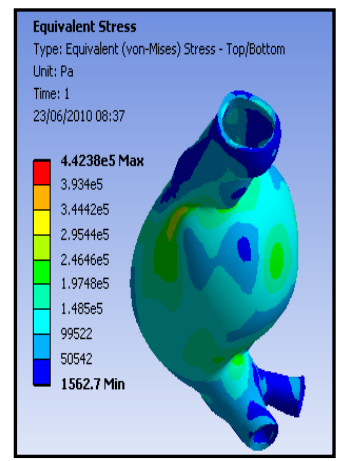

Figure 8. Stress distribution in the arterial wall obtained by finite element simulation.

\section{General}

At this point, it is important to highlight two aspects. The first one is that the accumulation of knowledge around the topic of accurate prediction of AAA rupture is large enough and significant advances have been achieved in last years although the physicians continue using the same criteria. The second one is related to the growing consensus that it is possible to improve the reliability of the AAA rupture assessment by means of the biomechanical approach.

Despite the growing interest for the behaviour of all these factors, many physicians question its clinical utility advocating the difficulties in its assessing during the everyday clinical practice. Often, these procedures require sophisticated software, very specific and accurate correlations and highly qualified personnel. This feeling appears clearly reflected in a survey carried out among vascular surgeons [85], whose outcomes are summarized in:

$90 \%$ of the institutions rely their rupture risk estimation on the maximum diameter and the expansion rate, whereas only $15 \%$ use the high mechanical stress criteria;

$40 \%$ of the institutions think that using their criteria, the rupture risk of AAAs is reliable in up to $75 \%$ of all cases; 
$18 \%$ of surveyed know and are familiar with the biomechanical criteria to estimate the aneurysm rupture risk, $63 \%$ know it but are not familiar with these criteria, where the other percentage has never heard about it.

Seems to be unlikely this knowledge replace the use of current criteria. Clinicians will always feel that large AAAs represent a rupture-threat and should be repaired. It is the small and medium size AAAs that could be examined by using these alternative diagnostic tools which, in the future, may prove to be useful adjunct to maximum diameter.

\section{Conclusions}

Aneurysmal disease and its progression is a very complex multifactorial process and its statistics are of great concern. The biomechanical approach here developed and substantiated can predict the rupture potential of a patient-specific AAA in any stage of evolution with sufficient accuracy to be clinically relevant. This predictive model is conceived by the integration of biological, morphological and structural information and can constitute a significant step in the clinical management of patients with aneurysm. Nowadays, we are developing a broader validation test of the proposed model by establishing its statistical significance with a large enough number of AAA cases.

\section{Author details}

Guillermo Vilalta* and Félix Nieto

Mechanical Engineering Division, CARTIF Centro Tecnológico, Boecillo (Valladolid), Spain

Enrique San Norberto and Carlos Vaquero

Angiology and Vascular Surgery Service,

University and Clinic Hospital of Valladolid, Valladolid, Spain

María Ángeles Pérez

ITAP Institute, University of Valladolid, Valladolid, Spain

José A. Vilalta

Industrial Engineering Department, Polytechnical University of Havana, Havana, 19340, Cuba

\section{References}

[1] Viceconti M, Taddei F, Van Sint Jan S, Leardini A, Critofolini L, Stea S, Baleani M (2008) MultiscaleModelling of the Skeleton for the Prediction of the Risk of Fracture. Clinical Biomechanics. 23: 845-852.

[2] Newman AB, Arnold AM, Burke GL, O'Leary DH, Manolio TA (2001) Cardiovascular Disease and Mortality in Older Adults with Small Abdominal Aortic Aneurysms Detected by Ultrasonography: the Cardiovascular Health Study. Annals of Internal Medicine. 134: 182-190.

${ }^{*}$ Corresponding Author 
[3] Johansson G, Swedenborg J (1986) Rupture Abdominal Aortic Aneurysms: A Study of Incidence and Mortality. Br. J. Surg. 73: 101-103.

[4] Karkos C, Mukhopadhyay I, Papakostas I, Ghost J, Thomson G, Hughes R (2000) Abdominal Aortic Aneurysm: the Role of Clinical Examination and Opportunistic Detection. Eur. J. Vasc. Endovasc Surg. 19: 299-303.

[5] Vorp DA (2007) Biomechanics of abdominal aortic aneurysm. J. Biomech. 40(9): 18871902.

[6] Blanchard JF, Armenian HK, Friesen PP (2000) Risk factors for abdominal aortic aneurysm: results of a case-control study. Am J Epidemiol. 151: 575-83.

[7] Tornwall ME, Virtamo J, Haukka JK, Albanes D, Huttunen JK (2001) Life-style factors and risk for abdominal aortic aneurysm in a cohort of Finnish male smokers. Epidemiology. 12: 94-100.

[8] Singh K, Bonaa KH, Jacobsen BK, Bjork L, Solberg S (2001) Prevalence of and risk factors for abdominal aortic aneurysms in a population-based study: the Tromso study. Am J Epidemiol. 154: 236-244.

[9] Bengtson H, Ekberg O, Aspdlin P, TakoLander R, Bergqvist D (1988) Aneurysmal disease: abdominal aortic dilatation in patients operated on for carotid artery stenosis. Acta Chit Scand. 143: 441-444.

[10] Anidjar S, Osborne-Pellegrin M, Coutard M, Michel JB (1992) Arterial hypertension and aneurismal dilatation. Kidney Int. 37 S61-66.

[11] Johansen K, Koepsell T (1986) Familial tendency for abdominal aortic aneurysms. JAMA. 256: 1934-1936.

[12] Tilson M (1985) Further studies of a putative crosslinking amino acid (3-deoxypyridinoline) in skin from patients with AAAs. Surgery. 98: 888-893.

[13] Kitchen ND (1989) Racial distribution of aneurysms in Zimbabwe. JR Soc Med. 82: 136145.

[14] Webster MW, Ferrel RE, St Jean FL, Majuroder et al (1991) Ultrasound screening of firstdegree relatives of patients with abdominal aortic aneurysm. J Vasc Surg. 12: 9-13.

[15] Kuivarilemi H, Tromp G, Prockop DJ (1991) Genetic causes of aortic aneurysms: unlearning at least part of what the textbooks say. J Clin Invest. 88: 1441-1445.

[16] Tromp G, Kuivaniemi H, Shikata H, Prockop DJ (1989) A single basemutation that substitutes serine for glycine 790 of the a 1 (III) chain of type III procollagen exposes an arginine and causes Ehlers-Danlos syndrome IV. J Biol Chem. 264: 1349-1356.

[17] Hinterseher I, Tromp G, Kuivaniemi H (2011) Genes and abdominal aortic aneurysm. Ann Vasc Surg. 25: 388-412.

[18] Nordon IM, Hinghliffe RJ, Holt PJ, et al (2009) Review of current theories for abdominal aortic aneurysm pathogenesis.Vascular. 17: 253-263.

[19] Shapira OM, Pakis S, Wassermann JP, Barzlllai N, Mashlah A (1990) Ultrasound screening for abdominal aortic aneurysms in patients with atherosclerotic peripheral vascular disease. J Cardiovasc Surg. 31: 170-175.

[20] Heistad DD, Marcus ML, Carsen GE et al (1981) Role of vasa vasorum in nourishment of the aortic wall. Am J Physiol. 240: 11781-11783.

[21] McMillan WD, Tamarina NA, Cipollone M (1997) Size matters: the relationship between MMP-9 expression and aortic diameter. Circulation. 96: 2228-2232. 
[22] Jagadesham VP, Scott DJ, Carding SR (2008) Abdominal aortic aneurysms: an autoimmune disease? TrensMol Med. 14: 522-529.

[23] Tanaka S, Komori K, et al (1994) Detection of active cytomegalovirus infection n inflammatory aortic aneurysms with RNA polymerase chain reaction. J Vasc Surg. 20: 235-243.

[24] Beckman EN (1986) Plasma cell infiltrates in atherosclerotic in abdominal aortic aneurysms. Am J ClinPathol. 85: 21-24.

[25] Newman KM, Jean-Claude J, et al (1994) Cellular localization of matrix metalloproteinases in the abdominal aortic aneurysm wall. J Vasc Surg. 20: 814-820.

[26] Nanda S, Sharma SG, Longo S (2009) Molecular targets and abdominal aortic aneurysms. Recent Pat Cardiovasc Drug Discov. 4: 150-159.

[27] Busuttil RW, Abou-Zamzam AM, Machleder HI (1980) Collagenase activity of the human aorta. A comparison of patients with and without abdominal aortic aneurysms. Arch Surg. 115: 1373-1378.

[28] Minion DJ, Davis VA, Nejezchleb PA, et al (1994) Elastin is increased in abdominal aortic aneurysms. J Surg Res. 57: 443-446.

[29] Vine N, Powell JT (1991) Metalloproteinases in degenerative aortic disease. Clin Sci. 81: 233-239.

[30] Thompson RW, Holmes DR, Mertens RA et al (1995) Production and localization of 92kilodalton gelatinase in abdominal aortic aneurysms. An elastolytic metalloproteinase expressed by aneurysm-infiltrating macrophages. J Clin Invest. 96: 318-326.

[31] Freestone T, Turner RJ, Coady A, et al (1995) Inflammation and matrix metalloproteinases in the enlarging abdominal aortic aneurysm. ArterioscThrombVasc Biol. 15: 1145-1151.

[32] Pyo R, Lee JK, Shipley JM, et al (2000) Targeted gene disruption of matrix metalloproteinase-9 (gelatinase B) suppresses development of experimental abdominal aortic aneurysms. J Clin Invest. 105: 1641-1649.

[33] Tamarina NA, McMillan WD, Shively VP, Pearce WH (1997) Expression of matrix metalloproteinases and their inhibitors in aneurysms and normal aorta. Surgery. 122: 264-272.

[34] Rizas KD, ippagunta N, Tilson MD (2009) Immune cells and molecular mediators in the pathogenesis of the abdominal aortic aneurysm. Cardiol Rev. 17: 201-210.

[35] Michel JB, Martin-Ventura JL, Egido J, et al (2011) Novel aspects of the pathogenesis of aneurysms of the abdominal aorta in humans. Cardiovasc Res. 90: 18-27.

[36] Yajima N, Masuda M, Miyazaki M, et al (2002) Oxidative stress is involved in the development of experimental abdominal aortic aneurysm: a study of the transcription profile with complementary DNA microarray. J Vasc Surg. 36: 379-385.

[37] Annambhotla S, Bourgeois S, Wang X, et al (2008) Recent advances in molecular mechanisms of abdominal aortic aneurysm formation. World J Surg. 32: 976-986.

[38] Sodeck G, Domanovits H, Khanakah G, et al (2004) The role of Chlamydia pneumoniae in human aortic disease-a hypothesis revisited. Eur J VascEndovasc Surg. 28: 547-552.

[39] Kilic A, Onguru O, Tugcu H, et AL (2006) Detection of cytomegalovirus and Helicobacter pylori DNA in arterial walls with grade III atherosclerosis by PCR. Pol J Microbiol. 55: 333-337. 
[40] Sakalihasan N, Pincemail J, et al (1996) Decrease of plasma vitamin E (alphaTocopherol) levels in patients with abdominal aortic aneurysm. Ann NY Acad Sci. 800: 278-282.

[41] Leung JH, Wright AR, Cheshire N, Crane J, Thom SA, Hughes AD, Xu Y (2006) Fluid structure interaction of patient specific abdominal aortic aneurysms: a comparison with solid stress models. BioMedical Engineering OnLine. http://www.biomedicalengineering-online.com/content/5/1/33. Accessed: 2011 Dec 21.

[42] Vorp DA, VandeGeest JP (2005) Biomechanical determinants of abdominal aortic aneurysm rupture. Arteriosclerosis, Thrombosis, and Vascular Biology. 25: 1558-1566

[43] Kleinstreuer C, Li Z (2006) Analysis and computer program for rupture-risk prediction of abdominal aortic aneurysms. BioMedical Engineering OnLine.Available: http://www.biomedical-engineering-online.com/content/5/1/19. Accessed: 2010 April 15.

[44] Vilalta G, Nieto F, Vaquero C, Vilalta JA (2010) Quantitative Indicator of Abdominal Aortic Aneurysm Rupture Risk Based on its Geometric Parameters, World Academy of Science, Engineering and Technology: 70: 181-185.

[45] Shum J, Martufi G, DiMartino E et al (2011) Quantitative assessment of abdominal aortic aneurysm geometry. [45] Ann. Biomed Eng. 39: 277-286. doi:10.1007/s10439-010-0175-3.

[46] Scotti, CM, Shkolnik AD, Muluk SC, Finol EA (2005) Fluid-structure interaction in abdominal aortic aneurysms: Effects of asymmetry and wall thickness. Biomedical Engineering OnLine.

http://www.biomedical-engineering-online.com/content/4/1/64.Accessed: 2012 Jan 21.

[47] Raghavan ML, Kratzberg J, de Tolosa EMC, et al (2006) Regional distribution of wall thickness and failure properties of human abdominal aortic aneurysm. J. Biomech. 39: 3010-3016.

[48] Frauenfelder T, Boutsianis E, Alkadhi H, Marincek B, Schertler T (2007) Simulation of blood flow within the abdominal aorta. Computational fluid dynamics in abdominal aortic aneurysms before and after interventions.Radiologe. 47: 1021-1028.

[49] M. Raghavan, D. Vorp, M. Federle, M. Makaroun, \& M. Webster, Wall stress distribution on three-dimensionally reconstructed models of human abdominal aortic aneurysm. Journal of Vascular Surgery, 31, 2000, 760-769.

[50] D. Wang, M. Makaroun, M. Webster, \& D. Vorp, Effect of intraluminal thrombus on wall stress in patient specific model of abdominal aortic aneurysm. Journal of Vascular Surgery, 3, 2002, 598-604.

[51] K. Wilson, A.J. Lee, P.R. Hoskins, F.G. Fowkers, C.V. Ruckley, \& A.W. Bradbury, The relationship between aortic wall distensibility and rupture of infrarenal abdominal aortic aneurysm. Journal of Vascular Surgery, 37, 2003, 112-117.

[52] Vilalta G, Nieto F, Vilalta JA et al (2012) Predicción del riesgo de ruptura de aneurismas de aorta abdominal. Método basado en los biodeterminantes geométricos. DYNA Ingeniería e Industria. 87: 66-73. doi: http://dx.doi.org/10.6036/4145.

[53] Georgakaratos E, Ioannou CV, Kamarianakis Y, Papaharilaou Y, Kostas T, Manousaki E, Katsamouris AN (2010) The role of geometric parameters in the prediction of abdominal aortic aneurysm wall stress. Eur. J. VascEndovasc Surg. 39: 42-48.

[54] Antigua L. Patient-Specific Modeling of Geometry and Blood Flow in Large Arteries. Ph.D thesis.Politecnico di Milano 2002 
[55] L. Antiga, B. Ene-Iordache, and A. Remuzzi (2003) Computational geometry for patient-specific reconstruction and meshing of blood vessels from MR and CT angiography. IEEE Transactions on Medical Imaging. 22: 674-684.

[56] Thomas JB, Antigua L, Che SL, Milner JS et al. Variation in the carotid bifurcation geometry of younger versus older adults. Stroke, 36: 2450-2456, 2005.

[56] Sacks MS, Vorp ML, RaghavanM;Federle MP, Webster MW (1999) In vivo threedimensional surface geometry of abdominal aortic aneurysms. Ann. Biomed. Eng. 27:469-479.

[57] Doyle BJ, Eng AC, Burke PE, et al (2009) Vessel asymmetry as an additional diagnostic tool in the assessment of abdominal aortic aneurysm. J. Vasc Surg. 49: 443-454.

[58] Swedenborg J, Eriksson P (2006) The intraluminal thrombus as a source of proteolytic activity. Ann. NY Acad. Sci. 1085: 133-138. doi: 10.1196/annals.1383.044.

[59] Vorp DA, Lee PC, Wang DH, et al (2001) Association of intraluminal thrombus in abdominal aortic aneurysm with local hypoxia and wall weakening. J. Vasc. Surg. 34: 291-299. doi: 10.1067/mva.2001.114813.

[60] Biasetti J, Gasser TC, Auer M, et al (2009) hemodynamics conditions of the normal aorta compared to fusiform and saccular abdominal aortic aneurysm with emphasize on thrombus formation. Ann. Biomed. Eng. 38: 380-390. doi: 10.1007/s10439-009-9843-6.

[61] Georgakarakos E, Ioannou CV, Volanis S et al (2009) The influence of intraluminal thrombus on abdominal aortic aneurysm wall stress. Int. Angiol. 28: 325-333.

[62] Bluestein D, Dumont K, De Beule M et al (2009) Intraluminal thrombus and risk of rupture in patient specific abdominal aortic aneurysm-FSI modeling. Compt. Meth. Biomech Eng. 12: 73-81.

[63] Georgakarakos E, Ioannou CV, Papahaliraou Y et al (2011) Computational evaluation of aortic aneurysm rupture risk: What have we learned so far?. J. EndovascTher. 18: 214225. doi: http://dx.doi.org/10.1583/10-3244.1.

[64] Doyle BJ, Corbett TJ, Callanan A (2009) An experimental and numerical comparison of the rupture locations of an abdominal aortic aneurysm. J. Endovasc. Ther. 16: 322-335.

[65] Darling RC, Messina CR, Brewster DC, et al (1977) Autopsy study of unoperated abdominal aortic aneurysm. The case for early resection.Circulation. 56 ( 3 suppl): II 161164.

[66] Soudah E, Vilalta G, Vilalta JA et al (2012) Idealized abdominal aortic aneurysm (AAA) geometry as predictor of hemodynamic stresses. Accepted for oral presentation in $6^{\text {th }}$ European Congress on Computational Methods in Applied Sciences and Engineering (ECOMAS 2012). Vienna Sep 10-14, 2012.

[67] Raghavan ML, Harbaugh RE (2005) Quantified aneurysm shape and rupture risk. J. Neurosurg. 102: 355-362.

[68] McGloughlin TM Doyle BJ (2010) New approaches to abdominal aortic aneurysm rupture risk assessment: engineering insight with clinical gain. Aterioscler. Thromb. Vasc. Biol. 30: 1687-1694.

[69] VandeGeest JP, Wang DHJ, Bohra A, Marakoun MS, Vorp DA (2006) A biomechanicsbased rupture potential index for abdominal aortic aneurysm risk assessment. Ann. N.Y. Acad. Sci. 1085: 11-21. 
[70] Li Z, Kleinstreuer C (2005) A new wall stress equation for aneurysm rupture prediction. An. of Biomedical Eng. 33: 209-213.

[71] Inzoli F, Boschetti F, Zappa M, Longo T, Fumero R (1993) Biomechanical factors in abdominal aortic aneurysm rupture. Eur. J. of Vasc. Surg. 7: 732-739.

[72] Mower WR, Buraff LJ, Sneyd J (1993) Stress distribution in vascular aneurysm: factors affecting risk of aneurysm rupture. J. of Surgical Research. 55: 1556-161.

[73] Vorp DA, Raghavan ML, Webster MW (1998) Stress distribution in aortic abdominal aneurysm: influence of diameter and symmetry. J. of Vasc Surg. 27: 632-639.

[74] He CM, Roach MR (1994) The composition and mechanical properties of abdominal aortic aneurysms. J. of Biomech Eng. 109: 298-304.

[75] Raghavan ML, Webster MW, Vorp DA (1996) Ex-vivo biomechanical behavior of abdominal aortic aneurysm: assessment using a new mathematical model. Ann Biomed Eng. 24: 573-582.

[76] Heng MS, Fagan MJ, Collier JW, Desai G, McCollum PT, Chetter IC (2008) Peak wall stress measurement in elective and acute abdominal aortic aneurysms. J. of Vasc Surg. 47: 17-22.

[77] González A, García de la Figal J (2009) Analysis of mechanical behavior of aorta section (in Spanish). IngenieríaMecánica. 12: 9-18.

[78] De Putter S, Wolters BJBM, Rutten MCM, Breeuwer M, Gerritsen FA, Van de Vosse FN (2007) Patient-specific initial wall stress in abdominal aortic aneurysm with a backward incremental method. J. of Biomech. 40: 1081-1090.

[79] Volokh KY, Vorp DA (2008) A model of growth and rupture of abdominal aortic aneurysm. J. of Biomech; 41: 1015-1021.

[80] Tong P, Fung YC (1976) The Stress-Strain Relationship for the Skin. J. of Biomech. 9: 649657.

[81] Rodríguez JF, Ruiz C, Doblaré M, Holzapfel GA (2008) Mechanical stresses in abdominal aortic aneurysm: influence of diameter, asymmetry, and material anisotropy. J. of Biomech Eng. 130: 256-267.

[82] Holzapfel GA, Gasser TC, Ogden RW (2000) A new constitutive framework for arterial wall mechanics and a comparative study of material models. J. of Elasticity. 61: 1-48.

[83] Papaharilaou Y, Ekaterinaris J A. Manousaki E, Katsamouris AN (2007) A decoupled fluid structure approach for estimating wall stress in abdominal aortic aneurysms. J. of Biomech. 40: 367-377.

[84] Vorp DA, VandeGeest JP (2005) Biomechanical determinants of abdominal aortic aneurysm rupture. Arteriosclerosis, Thrombosis and Vascular Biology. 25: 1558-1566.

[85] Vascops Vascular diagnostics. On-line survey: Clinical assessment of AAA rupture risk. Are biomechanical predictors needed? (2007). Last access: Sept-15-2010. Available in: http://www.vascops.com/files/survey2006.pdf. Accessed: 2011 March 24. 